\title{
How long and how much? Wait times and costs for initial private child mental health appointments
}

Melissa Mulraney*1,2, Cindy Lee*1,2, Gary Freed ${ }^{3}$, Michael Sawyer ${ }^{4,5}$, David Coghill ${ }^{1,2,6}$, Emma Sciberras ${ }^{1.2 .7}$, Daryl Efron ${ }^{1,2,6}$, Harriet Hiscock ${ }^{1,2,6}$

${ }^{1}$ Murdoch Children’s Research Institute, Melbourne, VIC, Australia

${ }^{2}$ Department of Paediatrics, University of Melbourne, Melbourne, VIC, Australia

${ }^{3}$ Department of Paediatrics, University of Michigan, Ann Arbor, MI, USA

${ }^{4}$ Women's and Children's Health Network, North Adelaide, SA, Australia

${ }^{5}$ Adelaide Medical School, University of Adelaide, Adelaide, SA, Australia

${ }^{6}$ Royal Children’s Hospital, Melbourne, VIC, Australia

${ }^{7}$ School of Psychology, Deakin University, Geelong, VIC, Australia

\section{Corresponding Author Information}

Corresponding author: Dr Melissa Mulraney, Health Services, Murdoch Children’s Research Institute (MCRI), 50 Flemington Road, Parkville, Melbourne, Vic. 3052, Australia. Fax: +61 39345 5900; email: melissa.mulraney@mcri.edu.au

\section{Acknowledgements}

This project was funded by a Project Grant from the Australian National Health and Medical

Research Council (NHMRC) (1129957). MCRI is supported by the Victorian Government's This is the author manuscript accepted for publication and has undergone full peer review but

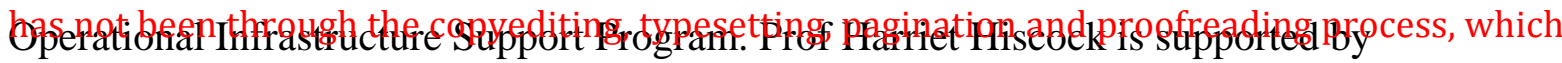
may lead to differences between this version and the Version of Record. Please cite this article an AHHMRG Pragtitiofnes Fellowship 1136222. Dr Sciberras is funded by an NHMRC Career 
Development Fellowship 1110688 (2016-2019) and a veski Inspiring Women’s Fellowship (2017-2019). Dr Efron is funded by a Clinician Scientist Fellowship from MCRI.

\section{Conflicts of interest}

The authors declare no conflicts of interest.

This article is protected by copyright. All rights reserved. 
How long and how much? Wait times and costs for initial private child mental health appointments

Melissa Mulraney*1,2 ${ }^{*}$ Cindy Lee*1,2 Gary Freed $^{3}$, Michael Sawyer ${ }^{4,5}$, David Coghill ${ }^{1,2,6}$, Emma Sciberras ${ }^{1.2 .7}$, Daryl Efron ${ }^{1,2,6}$, Harriet Hiscock $k^{1,2,6}$

${ }^{1}$ Murdoch Children’s Research Institute, Melbourne, VIC, Australia

${ }^{2}$ Department of Paediatrics, University of Melbourne, Melbourne, VIC, Australia

${ }^{3}$ Department of Paediatrics, University of Michigan, Ann Arbor, MI, USA

${ }^{4}$ Women's and Children's Health Network, North Adelaide, SA, Australia

${ }^{5}$ Adelaide Medical School, University of Adelaide, Adelaide, SA, Australia

${ }^{6}$ Royal Children’s Hospital, Melbourne, VIC, Australia

${ }^{7}$ School of Psychology, Deakin University, Geelong, VIC, Australia

\section{Corresponding Author Information}

Corresponding author: Dr Melissa Mulraney, Health Services, Murdoch Children’s Research Institute (MCRI), 50 Flemington Road, Parkville, Melbourne, Vic. 3052, Australia. Fax: +61 39345 5900; email: melissa.mulraney@mcri.edu.au

\section{Acknowledgements}


This project was funded by a Project Grant from the Australian National Health and Medical Research Council (NHMRC) (1129957). MCRI is supported by the Victorian Government's Operational Infrastructure Support Program. Prof Harriet Hiscock is supported by an NHMRC Practitioner Fellowship 1136222. Dr Sciberras is funded by an NHMRC Career Development Fellowship 1110688 (2016-2019) and a veski Inspiring Women’s Fellowship (2017-2019). Dr Efron is funded by a Clinician Scientist Fellowship from MCRI.

\title{
Conflicts of interest
}

The authors declare no conflicts of interest.

\begin{abstract}
Aim: To determine: 1) wait times and out-of-pocket costs for children attending private specialists for initial mental health appointments; and 2) whether these differed between specialists working in metropolitan versus rural areas and in low, medium and high socioeconomic areas.
\end{abstract}

Methods: Prospective secret shopper study whereby a researcher posed as a parent seeking an appointment for her child with anxiety or attention-deficit/hyperactivity disorder. We contacted 317 private paediatrician, psychiatrist and psychologist practices in Victoria and South Australia between 12 March and 5th May 2019.

Results: One third (29.8\%) of private practices were closed to new referrals. The average wait times for paediatricians, psychiatrists, and psychologists were 44, 41, and 34 days, 
respectively. Average out-of-pocket costs quoted were AU\$120 for paediatricians, AU\$176 for psychiatrists and AU\$85 for psychologists.

Conclusion: Parents face extensive wait times and substantial out-of-pocket costs when seeking private mental health services for their child.

Keywords: child, mental health, health services, costs, wait times 


\section{Brief points}

\section{What is already known on this topic?}

- Only 56\% of children and adolescents with a mental health disorder had accessed mental health care in 2013-14.

- Long wait times and high costs are the most common reasons given by parents for not accessing services with mental health professionals for their child.

\section{What this paper adds?}

- We provide the first objective Australian data on wait times and costs incurred by families seeking care for their child with mental health problems.

- Families face long wait times and substantial out-of-pocket costs when seeking an appointment with a paediatrician, psychiatrist or psychologist in the community. 
One in 7 (14\%) Australian children aged 4 to 17 years met criteria for a mental health disorder in 2013-14 (1). In Australia, approximately 3\% of children and adolescents with mental health problems are managed by the public health system (1) and these are typically the most complex cases. As such, most care is delivered privately. However, only $56 \%$ of children with a mental health disorder had accessed mental health services in 2013-14 (2). A systematic review of the literature identified a range of key barriers to parents accessing services for their child including: mental health literacy, stigma, familial circumstances and accessibility of services (3). In the most recent epidemiological survey of youth mental health in Australia, long wait times and high costs for appointments with mental health professionals were the two most common reasons given by parents for not accessing services (3). Despite this, the actual wait times and costs for child and adolescent mental health services in the private sector in Australia have not been quantified. An accurate understanding of these is essential for policy makers to ensure children and young people receive timely and affordable care $(4,5)$. Further, given that mental health disorders are more prevalent in rural than metropolitan regions (1) and in lower socioeconomic status (SES) than high SES areas (6, 7), and that there are fewer public services in rural versus metropolitan areas, understanding barriers to care in these areas is essential.

Children and adolescents with mental health problems most commonly attend general practitioners (GPs) (35\%) followed by private specialists including psychologists (24\%), paediatricians (21\%) and child and adolescent psychiatrists (7\%) for assessment and treatment of mental health problems (1). Given that GPs tend to see children for one visit only, most likely to organise a referral to a specialist (8), in this paper, we specifically 
consider services to specialists working in child mental health, i.e. paediatricians, child and adolescent psychiatrists and child and adolescent psychologists. In Australia, public services have no fee to the patient whereas private health care providers charge a fee which is subsidised by the federal government. In many cases the subsidy does not cover the total fee and thus patients will incur out-of-pocket costs (upfront cost minus the government rebate amount).

There is a scarcity of Australian data on wait times and costs for children to see a private mental health specialist. Kunin, Allen (9) found the average wait time for a general paediatric appointment in metropolitan Melbourne was 33 days with an out-of-pocket cost of \$128. However, in Kunin et al's study the appointments were for physical health complaints (i.e., a 3-year-old child with asthma, or an 8-month-old with eczema). Wait times to see a paediatrician for a mental health problem are typically reported to be longer than those for physical health problems (10). A number of studies (11-14) have reported wait times for child and adolescents mental health services, with wait times for child psychiatric consultations ranging from 8 (12) to 46 days (11). However, these studies report on countries with substantially different healthcare systems to Australia's.

We therefore aimed to determine the wait times and out-of-pocket costs for initial consultations with Australian private paediatricians, child and adolescent psychiatrists and psychologists for mental health services. Given that child mental health problems are more prevalent in rural Australia and in low SES areas (1) and that most psychiatrists and psychologists practice in major cities across Australia (15), we also aimed to compare costs 
and wait times between specialists working in rural versus metropolitan areas and across low, middle and high SES areas.

\section{Materials and Methods}

Sample

Lists of currently practising paediatricians, child and adolescent psychiatrists (hereafter referred to as psychiatrists) and child and adolescent psychologists (hereafter referred to psychologists) in Victoria and South Australia were generated. These specialist details were originally sourced from the Australian Paediatric Research Network (16), publicly-available specialty directory sites (for psychiatrists (17) and psychologists (18)) and online Google searches. These lists were used to generate a purposeful sample of paediatricians, psychiatrists and psychologists from both states with a range of remoteness and SES levels based on practice postcode. Specialists were excluded if online search details suggested they were subspecialists in a non-mental health field of paediatrics (e.g. paediatric allergists) or did not provide services to children. The population of Victoria is 6.7 million with 5.1 million people living in greater Melbourne $(19,20)$. Whilst the population of South Australia is 1.8 million with approximately 1.4 million people living in Adelaide (19, 20).

\section{Data collection}

A “secret shopper” method was used; a well-known and commonly used technique to determine the actual availability and patient cost of services $(21,22)$. A member of the study team (CL) made telephone calls to the private practices of the specialists posing as a parent 
seeking treatment for her child with a mental health problem. A mobile phone was used to make all calls so that the receptionists did not see the area code from where the call was being made. Telephone calls were made between $12^{\text {th }}$ March 2019 and $5^{\text {th }}$ May 2019. One of two randomly assigned clinical vignettes (see Supplementary material) were utilized to guide the telephone interaction. The vignettes were:

A. An 11-year-old girl with likely anxiety; and

B. A boy in Grade 2 with likely attention deficit hyperactivity disorder (ADHD)

These clinical vignettes were developed in consultation with practising paediatricians, psychologists and psychiatrists. The vignettes were deliberately designed to reflect core symptoms of anxiety and ADHD (the two most common mental health problems in childhood (1)) and to be 'average' referrals (i.e., were not designed to elicit responses to an urgent mental health problem). An attempt was made to book an appointment with a specific specialist from the list, informing clerical staff that there was a referral from a GP. Each call lasted approximately 4 minutes. The length of call varied as many receptionists requested additional details in which case the caller provided basic demographic information that matched the scenario in the vignette (e.g., male/female name of child and date of birth consistent with the child's age). If more detailed information was requested the caller deferred from answering citing a wish not to provide personal information until they had made a decision about booking an appointment.

The number of days (wait time) until the next available appointment time was obtained. For providers in group practices, if an appointment with the specified specialist was unable to be scheduled, the reason was noted and the time of the first available appointment with any other 
specialist at the practice was obtained. The out-of-pocket costs for an initial appointment were also recorded.

When an appointment time was offered, we deferred from accepting the appointment and stated that we would call back if the appointment was needed. No appointments were actually booked with any provider. There were no differences in wait times or costs according to which vignette was used and as such results are presented as a whole.

This study received ethics approval from the Human Research Ethics Committee at $<$ MASKED FOR PEER REVIEW $>$.

\section{Statistical analysis}

Descriptive measurements of the wait times and costs were determined using the statistical software package Stata Statistical Software Release 15. Using the postcode of private practices and the Australian Bureau of Statistics’ indexing tools (23), practice remoteness (Accessibility/Remoteness Index of Australia) and SES (Index of Relative Socioeconomic Disadvantage) were determined. Analyses of variance and t-tests were used to examine differences between the subsets of samples with the exception of paediatrician wait times where we present non-parametric test (Mann-Whitney and Kruskal-Wallis) results due to skewed data.

\section{Results}

Based on workforce proportions of specialists we aimed to survey 200 psychologists, 100 paediatricians, and 50 psychiatrists. Overall, 198 psychologists, 81 paediatricians, and 48 psychiatrists were contacted from Victoria and South Australia (see Figure 1). Thirty two per 
cent of psychologists, $46.7 \%$ of psychiatrists, and $15.2 \%$ of paediatricians were unable to offer an appointment, with a further $32.0 \%, 24.4 \%$ and $19.0 \%$ respectively, offering an appointment with an alternate specialist at the practice. The main reasons why the requested specialists (all three specialist types) were unable to offer an appointment were having too many current patients (25-31\%) and for psychologists and psychiatrists because they do not provide services to children of the age presented in the vignettes (22-28\%) (Table 1). The location (SES, rural/metropolitan) of participating clinics are reported in Table 2.

The median wait time was 44 days for paediatricians, 41 days for psychiatrists and 34 days for psychologists (Table 3). Although average wait times were longer in rural compared to metropolitan areas for psychologists and psychiatrists, these associations were nonsignificant. There were no associations between SES and wait times.

The median out-of-pocket fees quoted for an initial appointment were AU\$120, \$176 and \$85 for paediatricians, psychiatrists and psychologists, respectively (Table 4). Paediatrician and psychologist fees increased with an increasing SES gradient $(p<0.001)$. Additionally, metropolitan psychologists were more likely to report higher fees than rural psychologists ( $p$ $<0.001)$.

\section{Discussion}

The key finding from this study is the substantial number of clinicians for whom access was not possible at the time contact was made by the secret shopper, and the long wait times required to access child mental health specialists. One third (30\%) of private practices were 
closed to new referrals, and only $43 \%$ were able to offer an appointment with the requested clinician. The ability to offer an appointment was often limited because all three specialists types had reached their patient capacity or, for psychologists and psychiatrists, because they did not accept patients under the age of 12 years. This finding highlights the difficulties in accessing child mental health services and the inadequate workforce for younger children, particularly in rural areas (15).

Our data also highlight the substantial financial burden that parents of children with mental health problems experience. Depending on the specialist type, parents can expect to pay an out-of-pocket average of \$85-\$176 for an initial appointment. These estimated costs may differ to the actual costs incurred on the day of the appointment. The latter depend on the amount of time required to assess the child, the number of morbidities identified, and the requirement for follow-up consultations.

Our results show that the out-of-pocket costs for paediatricians and psychologists increase with an increasing SES gradient. This may be due to increased demand - higher income households are more likely to access care for their children (1) - and/or due to higher practice overhead expenses, such as rent which may drive up consultation costs. Whilst lower out-ofpocket costs charged by specialists practising in lower SES areas might facilitate access to care for low income families, the costs are such that they are likely to preclude access to ongoing care for these children.

Within the last 10 years, only one other secret shopper study has investigated wait times for private paediatricians in Australia (9). This study reported a 33-day average wait time for a 
child with a medical condition. However, consistent with our finding of a 44-day average wait time, paediatricians report that they usually see children with medical conditions sooner than children with developmental and behavioural conditions (10). The out-of-pocket costs for metropolitan private paediatricians do not appear to have increased in recent years.

This is the first study of its kind in Australia and our data collection covered two states in Australia and specialists practising in a range of remoteness and SES areas. Consequently, our findings are likely to generalise to the wider population of parents seeking help for their child's mental health problems. The study also has several limitations. Due to the nature of our secret shopper methodology, we could not take into account earlier appointment times that may be offered due to cancellations. Hence, the time to the next available appointment may be an overestimate. Despite this, the secret shopper method permits collection of real time data from a consumer's perspective. We did not collect data on the length of offered appointment which is likely to vary between professionals. Typically, psychologists and psychiatrists would have 50-60 minutes for an initial appointment, whereas for paediatricians this would usually be 30-45 minutes. Another limitation is that data were collected at only one point in time. It is not known whether there are seasonal changes in wait times to appointment. Anecdotally, clinicians report an increased demand for specialist child health services during the second half of the year when school submissions to the state education departments are due to apply for funding for additional learning support in the following year. We deliberately used non-urgent clinical vignettes so our findings may not generalise to urgent clinical referrals. Finally, we had fewer respondents from low SES and rural areas reflecting low numbers of specialists practising in these areas. 
Our study has important clinical implications. First, obtaining publicly available, accurate information on specialists' costs and scope of practice proved difficult as indicated by the number of clinicians we contacted who did not treat children below the age of 12 . This is a common barrier reported by parents aiming to seek or receive professional help for their child (1). Ensuring specialists provide accessible and accurate details about their practice contact and scope of practice (e.g. on professional body websites such as the RANZCP 'Find a psychiatrist' website) would be useful for parents. In addition, transparency of specialists’ fees would benefit parents and referring general practitioners. Parents could then make informed decisions about their willingness to pay for services.

Our findings can also inform policy makers in relation to Australian child and adolescent mental health systems. There is a dearth of child mental health specialists, especially for children under 12 years of age. Efforts to encourage training in the child mental health workforce are needed. This may include raising quotas and adding incentives for students to complete specialist training in child psychiatry (24). Additionally, enhanced mental health training for paediatricians, both in training and currently practicing, would be beneficial. Financial incentives and employment security for positions in areas of low supply should also be considered to tackle the likely undersupply in services in rural (24) and low SES regions. Removing the 'named clinician' requirement for Medicare referrals may lead to families being able to access care in a more timely manner.

Another consideration is the influence of public child and adolescent/youth mental health services on private services. Although there is a high volume of work conducted in the public child and adolescent/youth mental health services there is still a backlog of patients in the 
public system (24). This may be forcing families to seek help from the private sector. Thus, increased funding for public services could in turn improve availability of private mental health services. Additionally, if families are successful in receiving timely access to public services, they would avoid paying the substantial private out-of-pocket service costs.

This study focused on quantifying the wait times and costs for private child mental health specialists. Whilst there are no family out-of-pocket costs involved for accessing public child mental health services, future research should determine the wait times in the public sector for child mental health services. Additionally, variations in wait times across a full calendar year should be investigated to determine whether demand changes during the periods of school funding applications. Finally, in order to reduce out-of-pocket costs and wait times, consideration should be given to shared care models whereby GPs work with child mental health specialists to co-manage children with mental health problems. Such a model is used internationally and is being piloted in Queensland for the most common child mental health problem, ADHD, with initial promising results (25).

\section{Conclusion}

Families face long wait times and substantial out-of-pocket costs when seeking an appointment with a paediatrician, psychiatrist or psychologist in the community regardless of whether they live in rural or metropolitan areas. Whilst costs increased in line with SES of practice location the out-of-pocket costs in low SES areas are likely to be prohibitive for low income families. A larger and more equitably distributed child mental health workforce as 
well as increased patient Medicare reimbursement for child mental health services may be required to ensure timely, appropriate and affordable care for all. Developing and testing new models of care that increase accessibility and affordability is a priority. 


\section{References}

1. Lawrence D, Johnson S, Hafekost J, Boterhoven de Haan K, Sawyer M, Ainley J, et al. The mental health of children and adolescents: Report on the second Australian child and adolescent survey of mental health and wellbeing. Canberra: Department of Health; 2015. 2. Johnson SE, Lawrence D, Hafekost J, Saw S, Buckingham WJ, Sawyer M, et al. Service use by Australian children for emotional and behavioural problems: Findings from the second Australian Child and Adolescent Survey of Mental Health and Wellbeing. Australian New Zealand Journal of Psychiatry. 2016;50(9):887-98.

3. Reardon T, Harvey K, Baranowska M, O'Brien D, Smith L, Creswell C. What do parents perceive are the barriers and facilitators to accessing psychological treatment for mental health problems in children and adolescents? A systematic review of qualitative and quantitative studies. Eur Child Adolesc Psychiatry. 2017;26(6):623-47.

4. Gallucci G, Swartz W, Hackerman F. Impact of the wait for an initial appointment on the rate of kept appointments at a mental health center. Psychiatr Serv. 2005;56(3):344-6.

5. Westin AM, Barksdale CL, Stephan SH. The effect of waiting time on youth engagement to evidence based treatments. Community Ment Health J. 2014;50(2):221-8.

6. Reiss F. Socioeconomic inequalities and mental health problems in children and adolescents: a systematic review. Soc Sci Med. 2013;90:24-31.

7. Bøe T, Øverland S, Lundervold AJ, Hysing M. Socioeconomic status and children’s mental health: results from the Bergen Child Study. Soc Psychiatry Psychiatr Epidemiol. 2012;47(10):1557-66. 
8. Hiscock H, Mulraney M, Efron D, Freed G, Coghill D, Sciberras E, et al. Use and predictors of health services among Australian children with mental health problems: A national prospective study. Australian Journal of Psychology. 2019.

9. Kunin M, Allen AR, Nicolas C, Freed GL. Private general paediatric care availability in Melbourne. Aust Health Rev. 2017;41(1):63-7.

10. Hiscock H, Gulenc A, Efron D, Freed G. Inequity in Access to Paediatric Care for Developmental and Behavioural Versus Medical Problems in Australia: A National Survey. J Paediatr Child Health. 2018;54:705-6.

11. Steinman KJ, Shoben AB, Dembe AE, Kelleher KJ. How long do adolescents wait for psychiatry appointments? Community Ment Health J. 2015;51(7):782-9.

12. Bisgaier J, Polsky D, Rhodes KV. Academic medical centers and equity in specialty care access for children. Arch Pediatr Adolesc Med. 2012;166(4):304-10.

13. Cama S, Malowney M, Smith AJB, Spottswood M, Cheng E, Ostrowsky L, et al. Availability of outpatient mental health care by pediatricians and child psychiatrists in five US cities. Int J Health Serv. 2017;47(4):621-35.

14. Olin SCS, O’Connor BC, Storfer-Isser A, Clark LJ, Perkins M, Scholle SH, et al. Access to care for youth in a state mental health system: a simulated patient approach. J Am Acad Child Adolesc Psychiatry. 2016;55(5):392-9.

15. Australian Institute of Health and Welfare. Mental health workforce, Mental health services in Australia. Canberra, Australia: Australian Institute of Health and Welfare; 2018.

16. Australian Paediatric Research Network. [Available from: www.aprn.org.au. 
17. The Royal Australian \& New Zealand College of Psychiatrists. Find a Psychiatrist [Available from: www.yourhealthinmind.org/find-a-psychiatrist.

18. Australian Psychological Society. Find a Psychologist [Available from: www.psychology.org.au/Find-a-Psychologist.

19. Australian Bureau of Statistics. National, state and territory population. Canberra: Australian Bureau of Statistics; 2020.

20. Australian Bureau of Statistics. Regional population. Canberra: Australian Bureau of Statistics; 2020.

21. Bisgaier J, Rhodes K. Auditing access to specialty care for children with public insurance. N Engl J Med. 2011;364(24):2324-33.

22. Freed GL, Bingham A, Allen AR, Freed M, Sanci LA, Spike N. Actual availability of general practice appointments for mildly ill children. Med J Aust. 2015;203(3):145-.

23. Australian Bureau of Statistics. Socio-Economic Indexes for Areas (SEIFA) Technical Paper. Canberra, Australia: Australian Bureau of Statistics; 2008.

24. Victorian Auditor-General's Office. Child and youth mental health report 2019 [Available from: www.audit.vic.gov.au/report/child-and-youth-mental-health.

25. Newcomb D MP. Empowering General Practitioners to manage children with ADHD using the ECHO ${ }^{\circledR}$ model. International Journal of Integrated Care. 2018;18(s1).
Field Code Changed

Field Code Changed 
Table 1. Clinician reasons for unavailability

\begin{tabular}{lccc}
\hline Specialist type & Paediatrician & Psychiatrist & Psychologist \\
& $\mathrm{n}=27(\%)$ & $\mathrm{n}=32(\%)$ & $\mathrm{n}=116(\%)$ \\
\hline Too many patients & 25.9 & 25.0 & 31.0 \\
Does not treat age range & 3.7 & 28.1 & 22.4 \\
Sub-specialist & 11.1 & 9.4 & 2.6 \\
Other* & 33.3 & 9.4 & 21.6 \\
No reason given & 25.9 & 25.0 & 20.7 \\
\hline
\end{tabular}

*Includes: no longer working at practice, personal reasons, retired/retiring, on leave 
Table 2. Clinic locations

\begin{tabular}{|c|c|c|c|c|c|}
\hline \multirow[b]{2}{*}{ Specialist type } & \multicolumn{3}{|c|}{ Socioeconomic status (SES) $)^{\mathrm{a}}(\%)$} & \multicolumn{2}{|c|}{ Location $^{\mathrm{b}}(\%)$} \\
\hline & Low & Medium & High & Metropolitan & Rural \\
\hline \multicolumn{6}{|l|}{ Paediatrician } \\
\hline Victoria $(n=49)$ & 22.9 & 18.8 & 58.3 & 83.3 & 16.7 \\
\hline South Australia $(\mathrm{n}=32)$ & 16.1 & 32.3 & 51.6 & 87.1 & 12.9 \\
\hline \multicolumn{6}{|l|}{ Psychiatrist } \\
\hline Victoria $(n=31)$ & 9.7 & 25.8 & 64.5 & 80.7 & 19.4 \\
\hline South Australia $(n=14)$ & 0.0 & 21.4 & 78.6 & 100.0 & 0.0 \\
\hline \multicolumn{6}{|l|}{ Psychologist } \\
\hline Victoria $(n=97)$ & 7.4 & 33.7 & 59.0 & 71.6 & 28.4 \\
\hline South Australia $(\mathrm{n}=91)$ & 19.8 & 17.4 & 62.8 & 88.2 & 11.8 \\
\hline
\end{tabular}

a SES determined by Socio-Economic Indexes for Areas (SEIFA) decile rank. Low=1-4, Medium= 5-7, High= 8-10

${ }^{\mathrm{b}}$ Location based on Accessibility/Remoteness Index of Australia (ARIA) where: Metropolitan= Major cities, Rural= Inner regional, Outer regional, Remote 
Table 3. Wait time (days)

\begin{tabular}{|c|c|c|c|c|c|c|}
\hline Paediatrician & N (\%) & Median (IQR) & Range & $t / F^{a}$ & $\mathbf{d f}^{\mathrm{b}}$ & $\mathbf{p}$ \\
\hline Overall & 67 & $44(23-94)$ & $1-550$ & & & \\
\hline Location $^{c}$ & & & & -0.51 & & 0.61 \\
\hline Metropolitan & $58(87)$ & $46(23-81)$ & $1-550$ & & & \\
\hline Rural & 9 (13) & $31(28-111)$ & $21-229$ & & & \\
\hline $\mathrm{SES}^{\mathrm{d}}$ & & & & 0.60 & 2 & 0.74 \\
\hline Low & $12(18)$ & $32(23-81)$ & $21-550$ & & & \\
\hline Medium & $16(24)$ & $36(6-105)$ & $1-191$ & & & \\
\hline High & $39(58)$ & 49 (27-94) & $1-366$ & & & \\
\hline Psychiatrist & N (\%) & Median (IQR) & Range & & & $\mathbf{p}$ \\
\hline Overall & 22 & 41 (17-75) & $3-170$ & & & \\
\hline Location & & & & -0.40 & 20 & 0.69 \\
\hline Metropolitan & $18(82)$ & 30 (14-82) & $3-170$ & & & \\
\hline Rural & $4(18)$ & 66 (49-68) & $35-68$ & & & \\
\hline SES & & & & 0.36 & 2,19 & 0.70 \\
\hline Low & $2(9)$ & $34(4-63)$ & $4-63$ & & & \\
\hline Medium & $6(27)$ & $41(24-68)$ & $21-68$ & & & \\
\hline High & $14(64)$ & 44 (14-91) & $3-170$ & & & \\
\hline Psychologist & N (\%) & Median (IQR) & Range & $\mathbf{t} / \mathbf{F}^{\mathrm{a}}$ & $\mathbf{D f}^{\mathrm{b}}$ & $\mathbf{p}$ \\
\hline Overall & 123 & $34(8-56)$ & $0-201$ & & & \\
\hline Location & & & & -0.92 & 121 & 0.36 \\
\hline
\end{tabular}




\begin{tabular}{|c|c|c|c|c|c|c|}
\hline Metropolitan & $99(80)$ & $31(6-54)$ & $0-153$ & & & \\
\hline Rural & $24(20)$ & $51(21-58)$ & $0-201$ & & & \\
\hline SES & & & & 0.27 & 2,120 & 0.76 \\
\hline Low & $12(10)$ & $32(8-63)$ & $0-86$ & & & \\
\hline Medium & $33(27)$ & $35(13-54)$ & $0-201$ & & & \\
\hline High & 78 (63) & $33(7-63)$ & $0-153$ & & & \\
\hline \multicolumn{7}{|c|}{ a t-values are reported for location t-tests, F-values are reported for SES ANOVA } \\
\hline \multicolumn{7}{|c|}{${ }^{b} \mathrm{df}=$ degrees of freedom } \\
\hline \multicolumn{7}{|c|}{$\begin{array}{l}\text { ' SES determined by Socio-Economic Indexes for Areas (SEIFA) decile rank. Low= 1- } \\
\text { Medium }=5-7 \text {, High }=8-10\end{array}$} \\
\hline
\end{tabular}


Table 4. Out-of-pocket costs (AU\$) to families for initial appointment

\begin{tabular}{|c|c|c|c|c|c|c|}
\hline Paediatrician & N (\%)* & Median (IQR) & Range & $\mathbf{t} / \mathbf{F}^{\mathrm{a}}$ & $\mathbf{d f}^{\mathrm{b}}$ & $\mathbf{p}$ \\
\hline Overall & 71 & $120(98-162)$ & $0-253$ & & & \\
\hline Location $^{\mathrm{c}}$ & & & & 1.91 & 69 & 0.06 \\
\hline Metropolitan & $60(85)$ & $120(98-165)$ & $0-253$ & & & \\
\hline Rural & $11(15)$ & $100(75-140)$ & $0-141$ & & & \\
\hline $\mathrm{SES}^{\mathrm{d}}$ & & & & 9.70 & 2,68 & $<0.001$ \\
\hline Low & $14(20)$ & $84(60-130)$ & $0-148$ & & & \\
\hline Medium & $16(22)$ & $100(73-115)$ & $0-190$ & & & \\
\hline High & $41(58)$ & $132(100-183)$ & $57-253$ & & & \\
\hline Psychiatrist & N (\%)* & Median (IQR) & Range & $\mathbf{t} / \mathbf{F}^{\mathrm{a}}$ & $\mathbf{d f}^{\mathrm{b}}$ & $\mathbf{p}$ \\
\hline Overall & 25 & $176(155-200)$ & $0-415$ & & & \\
\hline Location & & & & -0.36 & 23 & 0.72 \\
\hline Metropolitan & $20(80)$ & $176(126-218)$ & $0-415$ & & & \\
\hline Rural & $5(20)$ & $192(180-200)$ & $175-246$ & & & \\
\hline SES & & & & 1.17 & 2,22 & 0.33 \\
\hline Low & $3(12)$ & $192(175-275)$ & $175-275$ & & & \\
\hline Medium & $6(24)$ & $190(155-246)$ & $155-415$ & & & \\
\hline High & $16(64)$ & $176(100-198)$ & $0-405$ & & & \\
\hline Psychologist & N (\%)* & Median (IQR) & Range & $t / F^{a}$ & $\mathbf{d f}^{\mathrm{b}}$ & $\mathbf{p}$ \\
\hline Overall & 124 & 85 (65-96) & $0-171$ & & & \\
\hline Location & & & & 4.38 & 122 & $<0.001$ \\
\hline
\end{tabular}




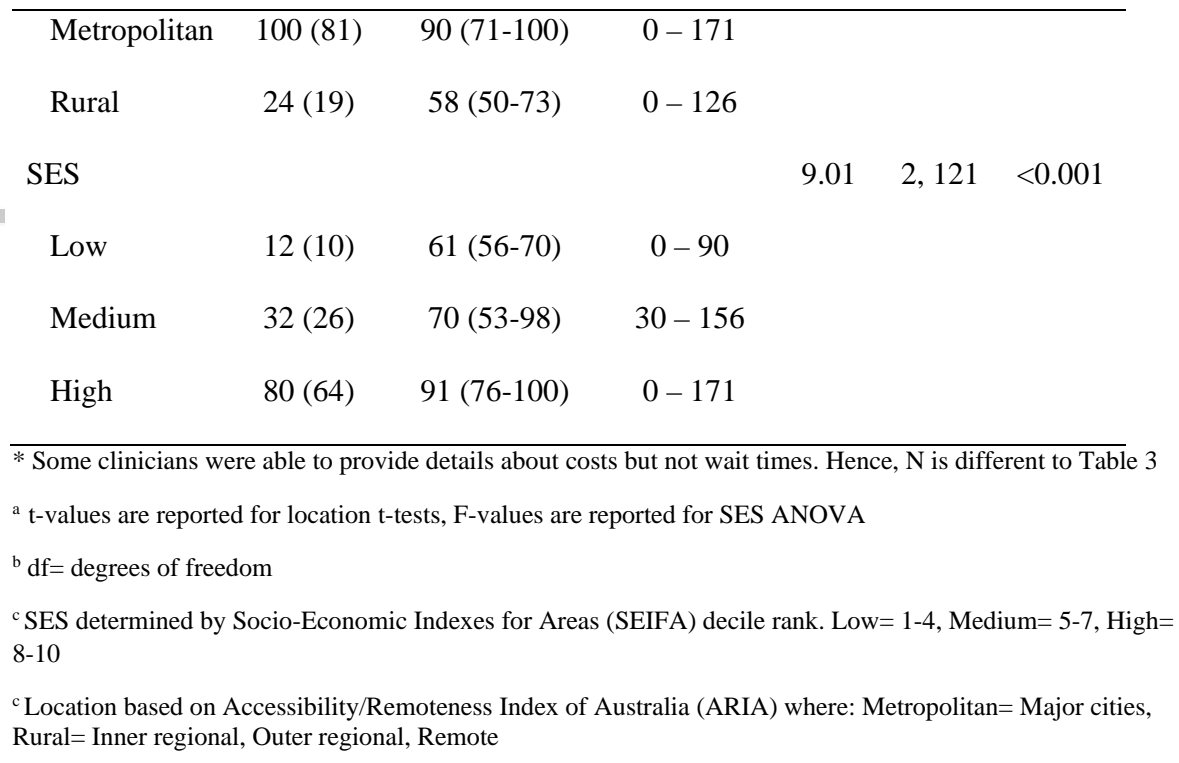


Figure 1: Phone calls conducted and appointment availability

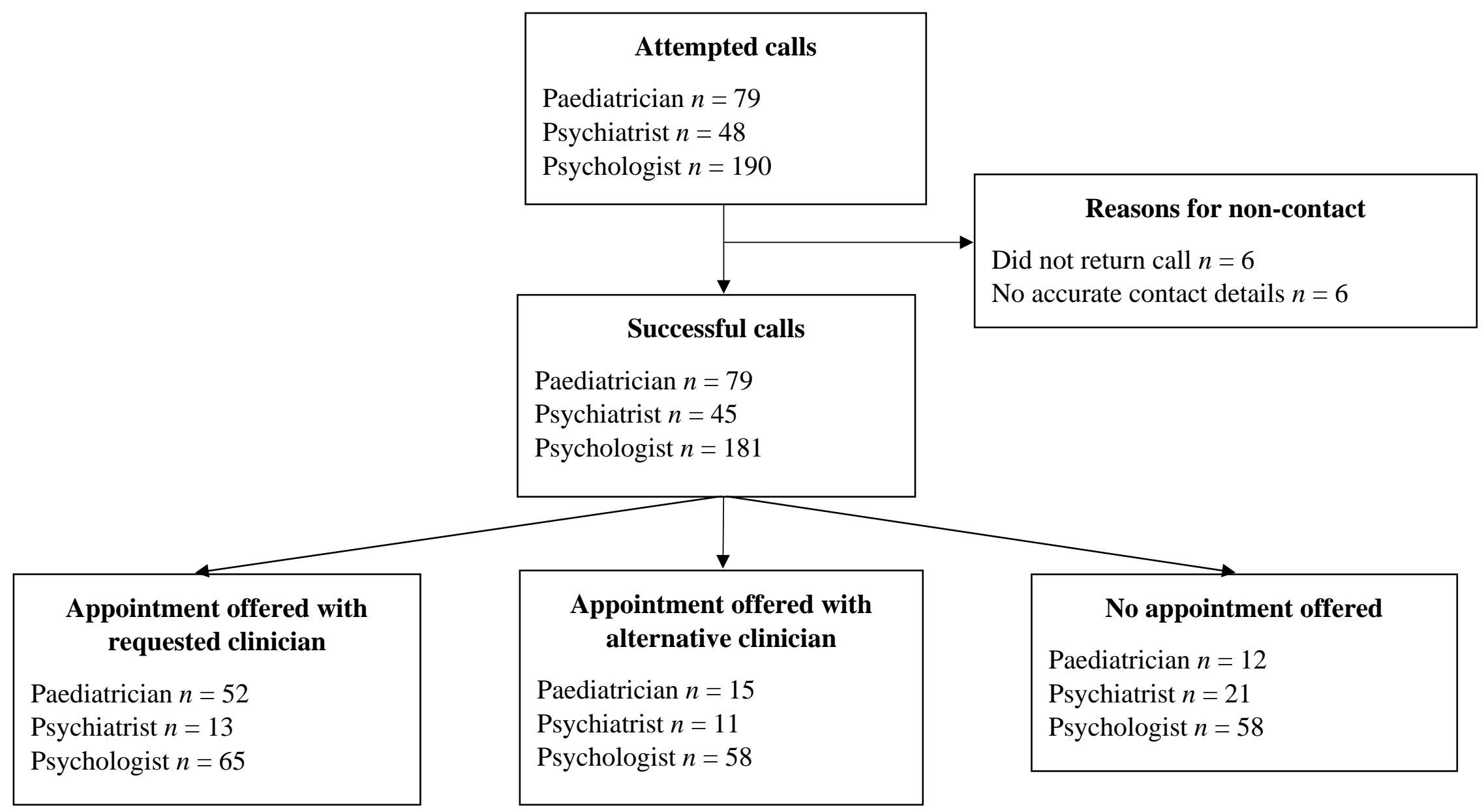

This article is protected by copyright. All rights reserved. 


\section{University Library}

\section{- M M N E R VA A gateway to Melbourne's research publications}

Minerva Access is the Institutional Repository of The University of Melbourne

Author/s:

Mulraney, M;Lee, C;Freed, G;Sawyer, M;Coghill, D;Sciberras, E;Efron, D;Hiscock, H

Title:

How long and how much? Wait times and costs for initial private child mental health appointments

Date:

2020-11-10

\section{Citation:}

Mulraney, M., Lee, C., Freed, G., Sawyer, M., Coghill, D., Sciberras, E., Efron, D. \& Hiscock, H. (2020). How long and how much? Wait times and costs for initial private child mental health appointments. JOURNAL OF PAEDIATRICS AND CHILD HEALTH, 57 (4), pp.526-532. https://doi.org/10.1111/jpc. 15253.

Persistent Link:

http://hdl.handle.net/11343/276599 